\title{
Bolletino dell' Associazione dei Musicologi italiani
}

Author(s): M. Guido Gasperini

Source: Bulletin de la Société française de musicologie, T. 2, No. 8 (Mar., 1921), p. 151

Published by: Société Française de Musicologie

Stable URL: http://www.jstor.org/stable/924836

Accessed: 30-04-2016 20:33 UTC

Your use of the JSTOR archive indicates your acceptance of the Terms \& Conditions of Use, available at

http://about.jstor.org/terms

JSTOR is a not-for-profit service that helps scholars, researchers, and students discover, use, and build upon a wide range of content in a trusted

digital archive. We use information technology and tools to increase productivity and facilitate new forms of scholarship. For more information about JSTOR, please contact support@jstor.org.

Société Française de Musicologie is collaborating with JSTOR to digitize, preserve and extend access to Bulletin de la Société française de musicologie 
la musique française, conférence faite au Foyer le 3 décembre 1913 ; Les Couperin (Revue hebdomadaire) ; Rameau (Courrier musical, 1908); Gluck (6 pages de La Pensée française, 1914) ; Berlioz, article de la Revue hebdomadaire, 1913 (regrettable); Emmanuel Chabrier (La Revue, 1911) : Massenet (Revue hebdomadaire, 1912); Debussy (Echo musical, 1914).

\section{Bolletino dell' Associazione dei Musicologi italiani, président} M. Guido Gasperini.

L'Associazione dei Musicologi italiani n'a pas perdu son activité pendant la guerre : elle vient d'en donner la preuve contraternelle à la Société française de musicologie en lui communiquant les fascicules, parus depuis 1917, de son catalogue général des œuvres musicales théoriques et pratiques conservées dans les bibliothèques d'Italie. Voici le détail de ce précieux dépouillement :

Séries VII et VIII : Ville de Modène, $R$. Biblioteca Estense, catalogue rédigé par M. Pio Lodi ; 14 facicules, - de juin 1916 à 1920.

Série IX : Ville de Ferrare, Biblioteca comunale. Rédigé par M. G. Agnelli; 1 fascicule. - mai 1917.

Série X : Ville de Naples, Archivio dell' Oratorio dei Filippini. Rédigé par M. Salvatore di Giacomo ; 4 fascicules, le $1^{\text {er }}$ consacré à une étude sur le Monastère dei Filippini, à Naples, "la Casa della Musica ", orné de diverses illustrations et reproductions. - 1918.

L'abondance de cette Bibliographie nous oblige à renvoyer au prochain Bulletin les comptes rendus des livres suivants et de quelques autres:

Encyclopédie de la musique et Dictionnaire du Conservatoire, vol. IV, Delagrave, 1920.

Lodis Schneider. - Claudio Monteverdi; L'Homme et son temps; le Musicien. 1 vol. in $8^{\circ}$, illustré. Librairie accadémique Perrin et $\mathrm{C}^{\mathrm{ie}}, 1921$.

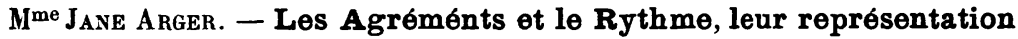
graphique dans la musique vocale française du XVIII ${ }^{\ominus}$ siècle 1 vol.gr. in $-8^{\circ}$, avec musique. Rouart, Lerolle et $\mathrm{C}^{\mathrm{ie}}, 1921$. 